Journal of Engineering and Applied Sciences 14 (11): 3777-3789, 2019

ISSN: 1816-949X

(C) Medwell Journals, 2019

\title{
Accurate Localization of Elderly People Based on Neural and Wireless Sensor Networks
}

\author{
Huda Ali Hashim, Salim Latif Mohammed and Sadik Kamel Gharghan \\ Department of Medical Instrumentation Techniques Engineering, \\ Electrical Engineering Technical College, Middle Technical University, Baghdad, Iraq
}

\begin{abstract}
This study aimed to localize the elderly while moving in a health care center or at home. Elderly localization was achieved by using a combination of the Received Signal Strength Indicator (RSSI) of Zigbee Anchor Nodes (ANs) and an artificial neural network. A Feed-Forward Neural Network (FFNN) was selected on the basis of the Levenberg-Marquardt (LM) training algorithm to train, test and validate data with the MATLAB Software. Two experiments were conducted in an indoor environment. The first and second experiments used three and four ANs, respectively. The effect of the numbers of ANs and neurons in each hidden layer of the FFNN on localization error was examined in terms of statistical analyses. Results show the better elderly localization accuracy achieved with four ANs compared with that obtained using three ANs. The four ANs achieved a localization error of $0.232 \mathrm{~m}$ (for testing) and improved by $65 \%$ compared with the three ANs. The results also reveal that the increase in the numbers of ANs and neurons can improve elderly localization accuracy. The second experiment (four ANs) provided a lower minimum localization error than the first experiment. Comparison of the results showed that our proposed method outperformed the other procedures in related literature in terms of localization error.
\end{abstract}

Key words: Accuracy, localization, MAE, neural network, wireless sensor network, Zigbee

\section{INTRODUCTION}

Localization techniques have attracted considerable interest in the medical field on the basis of different approaches such as Wireless Sensor Networks (WSN). Localization is one of the remarkable challenges in WSNs (Paul and Sato, 2017). Localization techniques in WSNs play a vital role in important applications such as person tracking, target tracking (Blumrosen et al., 2013; Ramiro Martinez-de Dios et al., 2017), monitoring, patient fall detection (Loet al., 2013), environmental management (Payal et al., 2015), wild forest areas (Zhao et al., 2013) and agriculture (Karim et al., 2015). For example, in sports, tracking techniques can provide important information about the movement or activity of body parts during motion. In medicine, a precise tracking technology that can be used in the homes of patients or at healthcare centers can analyze fall detection of patients or elderly people or be used for the rehabilitation of Parkinson's disease (Blumrosen et al., 2013; Zhang et al., 2012). In addition, various wireless medical applications obtain valuable information related to patient conditions (i.e., temperature, pressure, fall patient and heart pulse) when accompanied by technologies that determine patient location.
Given the various restrictions in WSN networks, accuracy is one of the challenges in WSN localization techniques (Halder and Ghosal, 2016). WSN techniques differ on the basis of; physical location (e.g., two and three-dimensional), types of environment (e.g., outdoor or indoor), network topology (e.g., centralized or decentralized remote sensing), type of wireless protocol (e.g., Bluetooth, Wi-Fi, Zigbee or RF) and cost of deployment. Consequently, substantial efforts have been devoted to improving localization accuracy to the centimeter scale. A battery powers the sensor nodes in WSN applications and the amount of energy consumed by the nodes determines the network lifespan of WSN applications. Therefore, low-power wireless protocols such as Zigbee or Bluetooth can be used for low-energy. Bluetooth coverage is limited to a smaller area compared with Zigbee. Wi-Fi consumes considerable power during transmission and reception (Gharghan et al., 2016a, b). The XBee S2C based on IEEE 802.15.4 protocol (Perera et al., 2017) possesses characteristics such as low power consumption within a suitable range, simple configuration, low cost, easy usage and high scalability (Hevrdejs et al., 2017), it is also self-configurable and contains a Received Signal Strength Indicator (RSSI) without adding any extra hardware. The RSSI

Corresponding Author: Sadik Kamel Gharghan, Department of Medical Instrumentation Techniques Engineering, Electrical Engineering Technical College, Middle Technical University, Baghdad, Iraq 
measurements using the Zigbee Anchor Node (AN) are appropriate for evaluating the localization or tracking of patients (Redondi et al., 2013).

Localization algorithms have become a substantial requirement to enhance WSN applications by determining the sensor node position with their absolute coordinates. Localization approaches can be divided into two types of techniques, namely, traditional localization and artificial intelligence techniques. Classical localization techniques can be further divided into two types: the range-based and range-free approaches (Gharghan et al., 2016a, b). The concept of range-based positioning relies on either an absolute point-to-point distance or directional information to obtain the location of sensor nodes. Range-based localization determines the distances or angles between two wireless sensor nodes. A disadvantage of this approach is that it often requires additional hardware (Payal et al., 2015). Examples of this approach include the Angle of Arrival (AoA) (Tomic et al., 2017), Time Difference of Arrival (TDOA) (Wang and $\mathrm{Wu}, 2017$ ), Time of Arrival (TOA) (Pak et al., 2017) and Global Positioning System (GPS) method (Liu et al., 2018).

The TOA method utilizes the concept of TOA of radio signal transmitting nodes. The disadvantages of this method include the difficulty that arises in calculating the time of flight in short distances. Establishing a highly accurate position system also presents a problem due to the challenges in handling time synchronization. TDOA method also uses time of flight but requires no time synchronization between the transmitter and receivers. However, this method requires time synchronization among receivers. TDOA also utilizes the concept of signal travel at different frequencies, indicating that signals travel at different speeds. Therefore, by measuring the difference in arrival time, the distance between the sensor nodes can be determined. However, many errors exist in the distance or location when TDOA and TOA algorithms are used as line-of-sight and multipath effect problems induced by building geometry and human body absorption, these issues result in technique complexity or ineffectiveness of the methods for indoor positioning systems.

GPS method depends on a series of satellites to determine localization. This method works well in outdoor environments but presents substantial limitations in indoor environments, these limitations include high cost and power consumption and the satellite's signal incapability to easily penetrate different types of physical barriers (i.e., windows, walls and chairs). Thus, GPS method is seldom used for indoor positioning systems (Luoh, 2014). The RSSI method relies on the signal strength transmitted. Previous studies have preferred this method for year owing to its low cost localization techniques (Zhao and Patwari, 2016) which require no extra hardware, an antenna array nor time synchronization (Mahapatra and Shet, 2018). This method uses a simple mathematical expression to express the localization relationships among WSNs. RSSI can also be used with Artificial Neural Networks (ANNs) (Azenha et al., 2012) due to the nonlinear properties of RSSI and ANN. Therefore, the RSSI $\mathrm{m}$ ethod is considered in this study.

The range-free approach features low accuracy in location detection of the wireless sensor node but is cost-effective. The approach relies on the connectivity among fixed nodes known as ANs and the stationary wireless sensor or another Mobile Node (MN). Examples of this method are weighted centroid localization (Kaur et al., 2017), centroid localization (Chen et al., 2017), hop-count-based localization (Sharma and Kumar, 2018) and pattern matching methods (Cheng et al., 2012). Hop-count method is easy to plan and perform because it can be implemented in a large wireless network. However, the localization error will increase. Pattern matching method is known for its popular fingerprint algorithm. The fingerprint algorithm features two phases, the first phase is called a radio map and receives signals at pre-defined positions that are recorded in an offline database (Li et al., 2016). The second phase uses a pattern-matching algorithm that uses an observed signal online with stored data to estimate the localization of an unknown node.

Artificial intelligence localization techniques, including ANN (Zhao et al., 2014; Zhi-kun and Zhong, 2015), Particle Swarm Optimization (PSO) (Zhao et al., 2014), Adaptive Neuro-Fuzzy Inference System (ANFIS) (Lin et al., 2014; Gharghan et al., 2018) and fuzzy logic (Velimirovic et al., 2012) have been employed in previous studies. Hybrid localization techniques such as PSO-ANN and Gravitational Search Algorithm (GSA) hybridized with ANN (GSA-ANN) (Gharghan et al., 2016a, b) have been adopted recently to minimize the localization error. To obtain an accurate prediction using an ANN Model, considerable data from various environments should be utilized in the training process. The model's performance will robustly depend on the selected input parameters. ANNs can filter out noise as desired in assessing localization algorithms and representing the complicated relationships among input data and output variables with the considerable knowledge directly obtained from these relationships.

The feed-forward algorithm is generally used with the back propagation training methodology which 
incorporates the standard gradient descent algorithm. Studies often employ Feed-Forward Neural Networks (FFNNs) that are unnecessarily complex (e.g., 3-4 hidden layers or numerous neurons) and the network size is occasionally neglected (Ostlin et al., 2010). Optimization of the learning processes of neural networks improves calculation by implementing the Levenberg-Marquardt (LM) algorithm which helps in direct calculation of the quasi-Hessian matrix and gradient vector without multiplying the Jacobian matrix nor creating more storage (Wilamowski and $\mathrm{Yu}, 2010$ ).

Localization models based on traditional localization techniques are computationally efficient but cannot achieve the desired accuracy. In recent year, ANNs have achieved more success than the previous localization techniques. ANNs are appropriate for filtering noise which cannot be easily and sufficiently characterized (Payal et al., 2015; Azenha et al., 2012). We propose a localization method that uses FFNN method on the basis of LM training algorithm to obtain prediction models that are more accurate and more computationally efficient than traditional empirical techniques. The proposed method measures the RSSI values from three Zigbee ANs to train, test and validate the FFNN. The number of ANs in the WSN and the neurons in each hidden layer of the FFNN were investigated to improve the accuracy of elderly localization. Our main contributions are summarized as follows:

- An accurate localization was achieved for elderly people on the basis of RSSI and the neural network in an indoor environment

- Localization error was investigated when the number of ANs of the WSN was increased

- Localization accuracy was confirmed when the number of neurons was increased in each hidden layer of the neural network

. The performance metrics of the proposed localization method was explored in terms of Mean Absolute Error (MAE)

. The proposed method outperforms previous techniques in terms of MAE relative to the comparative results

Literature review: Location prediction approach in WSNs has recently received substantial attention in research. In a study Thongpul et al. (2010), the location of a wireless sensor node was estimated on the basis of ANN and wavelet networks. The proposed method was used in an electrical laboratory and implemented and assessed in real indoor environments. The experimental results showed that the average location of $\mathrm{MN}$ error reached $0.9 \mathrm{~m}$. In harsh indoor environments such as non-line-of-sight and multipath effect, ANN approach was employed to predict the location of sensor nodes. In another research Nerguizian and Nerguizian (2007), the researchers proposed a fingerprinting technique by utilizing wavelet-based features that were extracted with ANN for a mobile station in an indoor environment. Two types of ANN algorithms were proposed, namely, the Multi Layer Perceptron (MLP) network and the generalized regression neural network. However, the system failed to achieve sufficient accuracy. The system requires improvement by increasing the number of coefficient indices, wavelet decomposition and number of training patterns. The experimental results showed that the average position error was $2 \mathrm{~m}$. One study, Azenha et al. (2012) presented the localization of indoor quasi-structured environments based on MLP network method. The system utilizes hardware on the basis of the Zigbee (CC2431) chip. The dimension of a training grid was $5 \times 5 \mathrm{~m}$ and approximately 55 points were employed within the testing area. The experimental results showed that the average error was $2 \mathrm{~m}$.

Localization approaches were recently achieved on the basis of artificial intelligence. Gogolak et al. (2013) used a FFNN type on the basis of LM training algorithm to predict the position of MNs. The experiment has been conducted in an indoor environment. The $\mathrm{MN}$ accumulated the RSSI samples of five ANs. The RSSI data were utilized in training the neural network. The experiment used a Zigbee wireless protocol in its WSN. The researchers compared their results with another method called Weighted k-Nearest Neighbor (WkNN). The experimental study showed that the localization accuracy of WkNN approach based on five ANs is better than the ANN technique whereas the accuracy of ANN is better than that of WkNN with three ANs. By Brunato and Battiti (2005), a statistical learning theory for location fingerprinting is presented. The theory utilizes IEEE802.11b, a Wi-Fi standard based on support vector machine. The estimated location relies on the RSSI measurement approach. Low algorithmic complexity has been employed as the suitable technique. However, high localization error was recorded. The experimental results showed that the average localization error of $3.04 \mathrm{~m}$. By Hwang et al. (2011), the researhers presented an indoor localization technique based on ANN. The RSSI sensed by the Zigbee/IEEE 802.15.4 module was utilized to determine the object location. Simulation results revealed that the proposed ANN technique yielded high accuracy, although, the RSSI sensed was unstable and the accuracy of the polar form was better than that of the rectangular form. 
Gharghan et al. (2018) used ANFIS to estimate the location of a bicycle while moving on a cycle track. The researchers utilized the RSSI of the Zigbee wireless protocol (i.e., XBee S2C) using three ANs to train ANFIS in indoor and outdoor environments. The distance between the moving bicycle $(\mathrm{MN})$ and $\mathrm{AN}$ which was located in the center of the cycle track was estimated. Based on the ANFIS technique, a $0.283 \mathrm{~m}$ distance error was obtained in an indoor environment. Gharghan et al. (2016a, b) used the hybrid technique, GSA-ANN, to evaluate the distance between the moving bicycle on the track and the $\mathrm{AN}$ that was positioned in the center of the cycle track. The RSSI of the Zigbee was used to train, test and validate the ANN. GSA optimization algorithm was used to select the optimum value of the learning rate and neurons in each hidden layer of ANN to obtain the minimum distance error. Consequently, the distance estimation error improved to $0.2 \mathrm{~m}$ in an indoor environment. By Gharghan et al., (2016a, b), PSO-ANN was used in cycling application. The RSSI of XBee S2C was used to train ANN and accurately determine the distance between $\mathrm{MN}$ (i.e., bicycle) and AN. The hybrid method accomplished minimum distance error in an indoor environment wherein an estimated distance of $0.208 \mathrm{~m}$ was achieved.

Another study Li et al. (2016) adopted PSO-ANN to improve the localization accuracy in indoor environments using WiFi technology. The researchers proposed an indoor localization scheme employing the PSO-ANN and "Affinity Propagation (AP) clustering algorithm". The combination between PSO and ANN introduced less time consuming and provided faster convergence as well as high learning and estimation speed. The estimation performance of the PSO-ANN is compared relative to the common widely used methods: Backpro Pagation (BP) ANN and k-Nearest Neighbor (kNN). The results disclosed that the PSO-ANN outperformed the BP-ANN and $\mathrm{kNN}$ in terms of localization error by 8 and $24 \%$. Where the PSO-ANN achieved a localization error of $1.893 \mathrm{~m}$. Channel State Information (CSI) of WiFi based Convolutional Neural Network (CNN) for indoor localization is proposed by Chen et al. (2017). The proposed method, namely, ConFi algorithm. The ConFi has two phases: training phase and localization phase. In the former phase (i.e., training), the CSI is gathered from reference points and employed for training the CNN through "stochastic gradient descent algorithm". In the later phase, the CSI of the target is applied to the CNN and the localization is computed as the weighted centroid of the reference points with high outcome rating. The authors compared the mean localization error of $\mathrm{ConFi}$ with Deep ANN and DeepFi (Wang et al., 2017). The ConFi optimized the mean localization error by 21.64 and
9.2\% relative to the Deep ANN and DeepFi, respectively. However, the ConFi presented a localization error of $1.3654 \mathrm{~m}$.

The location accuracy of WSNs is essential for planning wireless medical applications related to low power consumption in WSN's. Based on previous works that used artificial intelligence techniques or algorithms, the localization accuracy remains unsatisfactory for several of these works. Consequently, in terms of an accurate localization, a challenge exists in indoor environments where in the estimated error must be decreased from several meters to some centimeters. The challenge is caused by the presence of obstacles (door, windows and movements of people) which cause attenuations or fluctuations in the RSSI and which will produce errors in localization estimation. Therefore, the previous short comings have motivated us to employ an FFNN type on the basis of LM training algorithm for the improvement of the localization error of the elderly while moving in indoor environments based on the measurement of RSSI of ANs in WSNs. We also studied and analyzed the effect of increasing the number of ANs and neurons in each hidden layer of the neural network on the localization accuracy in this research.

\section{MATERIALS AND METHODS}

Two experiments were conducted to measure the RSSI of ANs. The first and second experiments, used 3 and 4 Zigbee ANs, respectively. Both experiments utilized the measured RSSI values to train, test and validate the FFNN.

Experiment setup: In this study, two experiments were conducted in the Electronic Lab in the Electrical Engineering Technical College, Iraq. The lab was selected due to its large available area. Figure 1 shows the layout of the WSN and the distributed ANs and $\mathrm{MN}$ in an indoor environment for elderly localization. The lab featured an area of $14 \times 6.85 \mathrm{~m}$ with a free area of $8.6 \times 3.23 \mathrm{~m}$ considered for testing. Accordingly, 33 locations were used for RSSI measurements and are represented by round points in Fig. 1. The predefined locations were divided into 3 groups with 3 columns and 11 rows. The first group, A1-A11, occupies the top portion of Fig. 1; the second group, $\mathrm{B} 1-\mathrm{B} 11$, lies in the middle; the third group, $\mathrm{Cl}-\mathrm{C} 11$, is found at the bottom of figure. The distance between points or grid resolution was selected at $0.8 \mathrm{~m}$ to obtain more test points. The ANs (AN1-AN4) were located $2.5 \mathrm{~m}$ away from the ground and near the ceiling of the lab (Fig. 1). The $\mathrm{MN}$ was worn by a person moving along the predefined position points to collect 


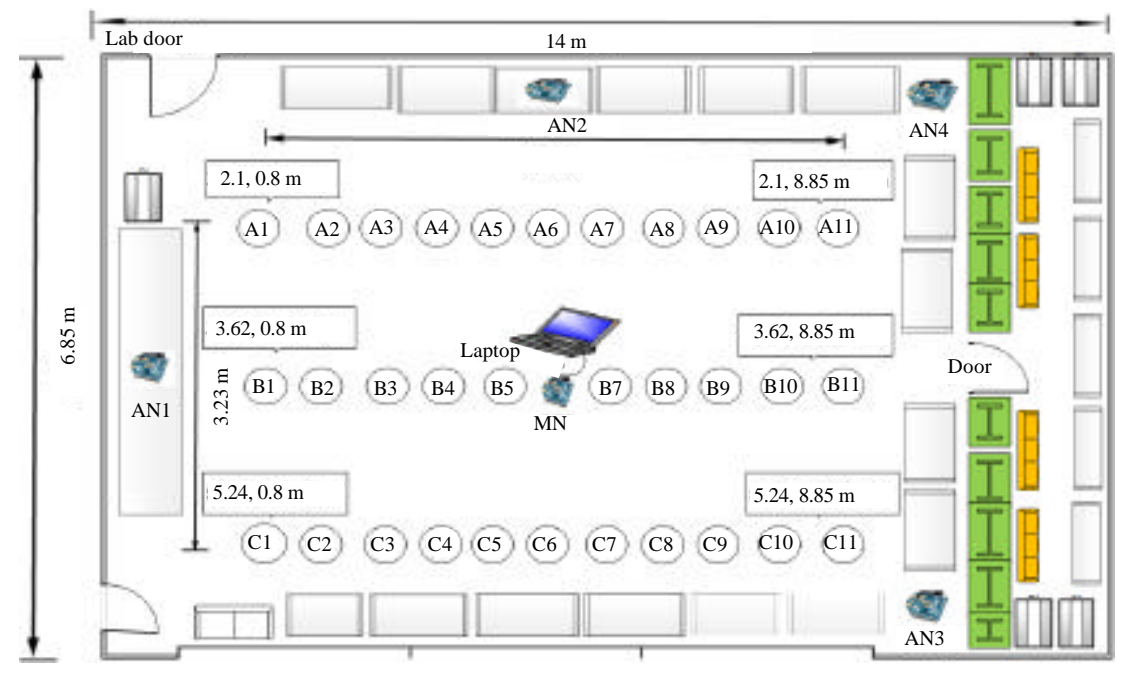

Fig. 1: Layout of the two experiments in the electronic lab

the RSSI values from the Ans. In the first and second experiments, the MN obtained RSSI from three (AN1-AN3) and four ANs (AN1-AN4), respectively. The number of ANs was increased to 4 in the second experiment to increase the elderly localization accuracy. The experiments were conducted in similar environmental conditions in the lab which contains electronic boards for student teaching, measurement devices, PCs and pieces of furniture such as chairs and table.

RSSI measurements: RSSI was measured for 33 predefined points in the testing area of the lab. Measurements of these points or locations (i.e., $\mathrm{x}$ and $\mathrm{y}$-axis) were determined on the basis of distance in meters (Table 1). The tested points were located within the testing area. The Zigbee MN moved along the 33 predefined positions and collected 100 RSSI samples from each AN at $1 \mathrm{~s}$ for each sample. Each sample contained 20 bytes of data packets. The ANs transmitted the packets to the $\mathrm{MN}$. The $\mathrm{MN}$ recorded the RSSI values using a laptop with X-CTU Software (Erazo-Rodas et al., 2018). The XCTU Software was adjusted to record 300 RSSI samples at each position from three ANs (100 samples for each AN) for the first experiment and 400 RSSI samples (100 samples for each $\mathrm{AN}$ ) for the second experiment which used four ANs. Accordingly, 9,900 and 13,200 RSSI samples were measured and recorded for the first (three ANs) and second experiments (four ANs), respectively. A total of 100 RSSI values were adopted to reduce the effect of signal impairments such as equipment and furniture, present in the electronic lab. Radio signals are extremely sensitive to variations and reflections in indoor environments. Thus, the equipment, tables,
Table 1: Positions of 33 points in the electronic lab

\begin{tabular}{lll}
\hline & Position (m) & \\
Groups & $-\mathrm{x}$ & $\mathrm{y}$ \\
\hline First group & & \\
A1 & 2.10 & 0.80 \\
A2 & 2.10 & 1.60 \\
A3 & 2.10 & 2.40 \\
A4 & 2.10 & 3.22 \\
A5 & 2.10 & 4.30 \\
A6 & 2.10 & 4.83 \\
A7 & 2.10 & 5.64 \\
A8 & 2.10 & 6.44 \\
A9 & 2.10 & 7.25 \\
A10 & 2.10 & 8.50 \\
A11 & 2.10 & 8.86 \\
Second group & & \\
B1 & 3.62 & 0.80 \\
B2 & 3.62 & 1.60 \\
B3 & 3.62 & 2.40 \\
B4 & 3.62 & 3.22 \\
B5 & 3.62 & 4.30 \\
B6 & 3.62 & 4.83 \\
B7 & 3.62 & 5.64 \\
B8 & 3.62 & 6.44 \\
B9 & 3.62 & 7.25 \\
B10 & 3.62 & 8.50 \\
B11 & 3.62 & 8.86 \\
Third group & & \\
C1 & 5.24 & 0.80 \\
C2 & 5.24 & 1.60 \\
C3 & 5.24 & 2.40 \\
C4 & 5.24 & 3.22 \\
C5 & 5.24 & 4.30 \\
C6 & 5.24 & 8.63 \\
C7 & 5.24 & 8.54 \\
C8 & 5.24 & \\
C9 & 5.24 & \\
C10 & 5.24 & \\
C11 & 5.24 & \\
\hline & & \\
\hline
\end{tabular}

windows and doors in the lab affected the accuracy of localization. Figure 1 shows the 3,300 samples of RSSI 


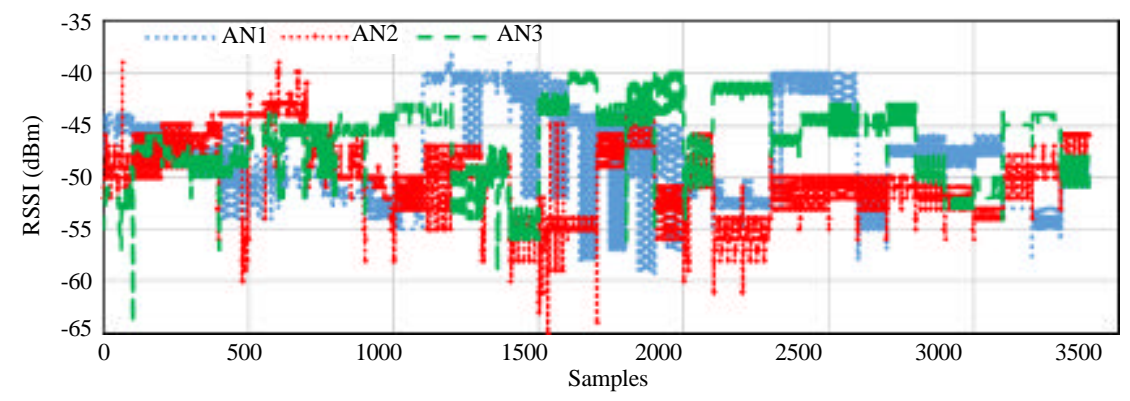

Fig. 2: First experiment: measured RSSI values at the MN for three ANs

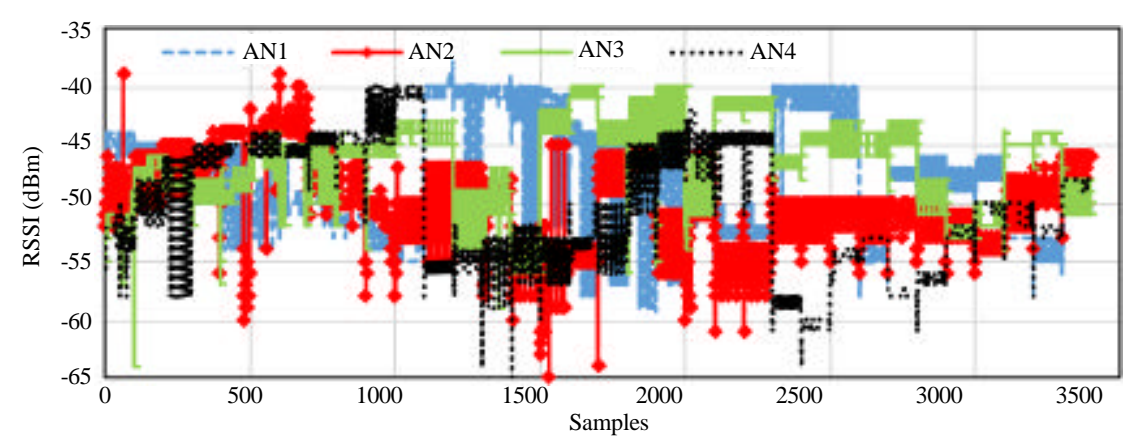

Fig. 3: Second experiment: measured RSSI values at the MN for four ANs

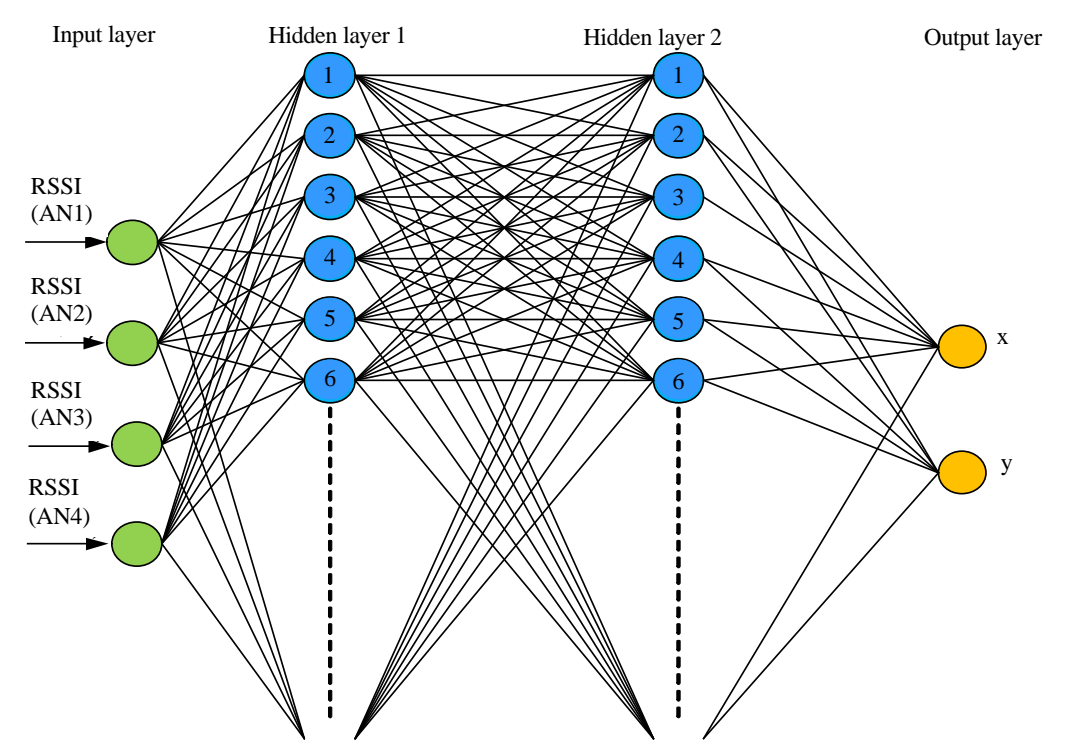

Fig. 4: Architecture of ANN algorithm

values collected from three Ans whereas Fig. 4 shows another 3,300 samples of RSSI values were collected four ANs. Figure 1-4 show the fluctuation of RSSI values due to signal impairments in indoor environments.

Neural network for elderly localization: In this study, an FFNN type based on LM training algorithm was employed to train, test and validate the RSSI measurements for estimation of elderly location. The location was determined by the coordinates of the $\mathrm{x}$ and $\mathrm{y}$-axis. ANN was adopted to achieve minimum localization error within a few centimeters. FFNN was implemented using MATLAB Software 2018. The LM training algorithm was selected because it can achieve minimum 
Table 2: Designed parameters of FFNN

\begin{tabular}{lll}
\hline Parameters & Values & Descriptions \\
\hline Number of inputs for first experiment & 3 & Three ANs \\
Number of inputs for second experiment & 4 & Four ANs \\
Number of outputs & 2 (x and y) & Linear activation function \\
Number of hidden layer & 2 & Four cases \\
Number of neurons in hidden layer1 & $5,10,20,40$ & Four cases \\
Number of neurons in hidden layer2 & $5,10,20,40$ & ------ \\
Learning rate & 0.7 & --- function $^{---}$ \\
Epoch & 1000 & 9,900 for three ANs \\
Target error & $1 \times 10^{-3}$ & 13,200 for four ANs \\
Collected RSSI for first experiment & 3,300 for each AN &
\end{tabular}

localization error as proven by Gharghan et al. (2016a, b). The LM algorithm is an efficient and rapidly functioning algorithm that mainly achieves flexible training. This algorithm also performs quadratic approximation to hasten the training procedure Wilamowski and Yu (2010). The LM algorithm was tested and used to solve for function approximations of different problems. LM algorithm has been proven to train ANNs faster (depending on the problem) than that of the standard gradient descent back propagation algorithm (Pradityo et al., 2017). When applying FFNN to solve a specific problem, the number of inputs and outputs, the number of hidden layers, the number of neurons in each hidden layer, learning rate, population size and the type of activation functions used in the neurons are remarkable parameters that must be determined before training, testing and validating the process. Table 2 shows the designed parameters of FFNN in our study. The number of inputs is based on ANs wherein the number of inputs was 3 for the first experiment, that is RSSI 1 for AN1, RSSI 2 for AN2 and RSSI 3 for AN3 and four for the second experiment that is RSSI 1 for AN1, RSSI 2 for AN2, RSSI 3 for AN3 and RSSI 4 for AN4. The output refers to the location ( $x$ and $y$-axis) of $\mathrm{MN}$ while moving on the predefined locations inside the testing area.

The input and output of FFNN for indoor environments are expressed as Eq. 1 and 2, respectively where RSSI, denotes the RSSI of kth AN for the jth sample in Eq. 1. The $\mathrm{x}$ and $\mathrm{y}$-axis represent the position of the elderly inside the testing area and $\mathrm{m}$ indicates the entire number of RSSI samples in Eq. 2. A total of 9,900 (first experiment) and 13,200 (second experiment) RSSI samples were utilized for the elderly localization experiments. The samples for the first experiment were gathered from three ANs whereas those for the second experiment were collected from four ANs. Among the measured RSSI values, $70 \%$ were used for training and $30 \%$ for testing and validating (15\% for testing and $15 \%$ for validating) FFNN:

$$
\begin{gathered}
\text { Input }=\left[\begin{array}{cccc}
\operatorname{RSSI}_{11} & \mathrm{RSSI}_{12} & \mathrm{RSSI}_{13} & \mathrm{RSSI}_{14} \\
\operatorname{RSSI}_{21} & \mathrm{RSSI}_{22} & \mathrm{RSSI}_{23} & \mathrm{RSSI}_{24} \\
\vdots & \vdots & \vdots & \\
\operatorname{RSSI}_{\mathrm{m} 1} & \mathrm{RSSI}_{\mathrm{m} 2} & \mathrm{RSSI}_{\mathrm{m} 2} & \mathrm{RSSI}_{\mathrm{m} 4}
\end{array}\right] \\
\text { Output }=\left[\begin{array}{cc}
\mathrm{x}_{11} & \mathrm{y}_{12} \\
\mathrm{x}_{21} & \mathrm{y}_{22} \\
\vdots & \vdots \\
\mathrm{x}_{\mathrm{m} 1} & \mathrm{y}_{\mathrm{m} 2}
\end{array}\right]
\end{gathered}
$$

Two hidden layers were selected to obtain the minimum localization error. One hidden layer was excluded from this study because it failed to provide high localization accuracy on the basis of our trial-and-error test. Neurons were trained (i.e., 5, 10, 20 and 40), tested and validated in each hidden layer to obtain minimum localization error. A total of 30 neurons were excluded in our results because they yielded similar results with another set of 20 neurons after training, testing and validating the neural network. The selection of learning rate, epoch and target error was subjected to the trial-and-error process.

\section{RESULTS AND DISCUSSION}

Implementation of FFNN algorithm: After gathering the measured RSSI samples, the FFNN was trained, tested and validated in two scenarios whereby the first and second scenarios used three ANs (9,900 samples) and four ANs (13,200 samples), respectively, to show the performance of FFNN algorithm. The simulation results were correspondingly achieved. Each scenario included four cases (i.e., 5, 10, 20 and 40 neurons) of the 2 hidden layers for three and four ANs as shown in Fig. 5 and 6, respectively. The neurons for each hidden layer were examined to determine the neurons that can yield the minimum localization error. Figure 5a-d present the results after training, testing and validating the FFNN algorithm 

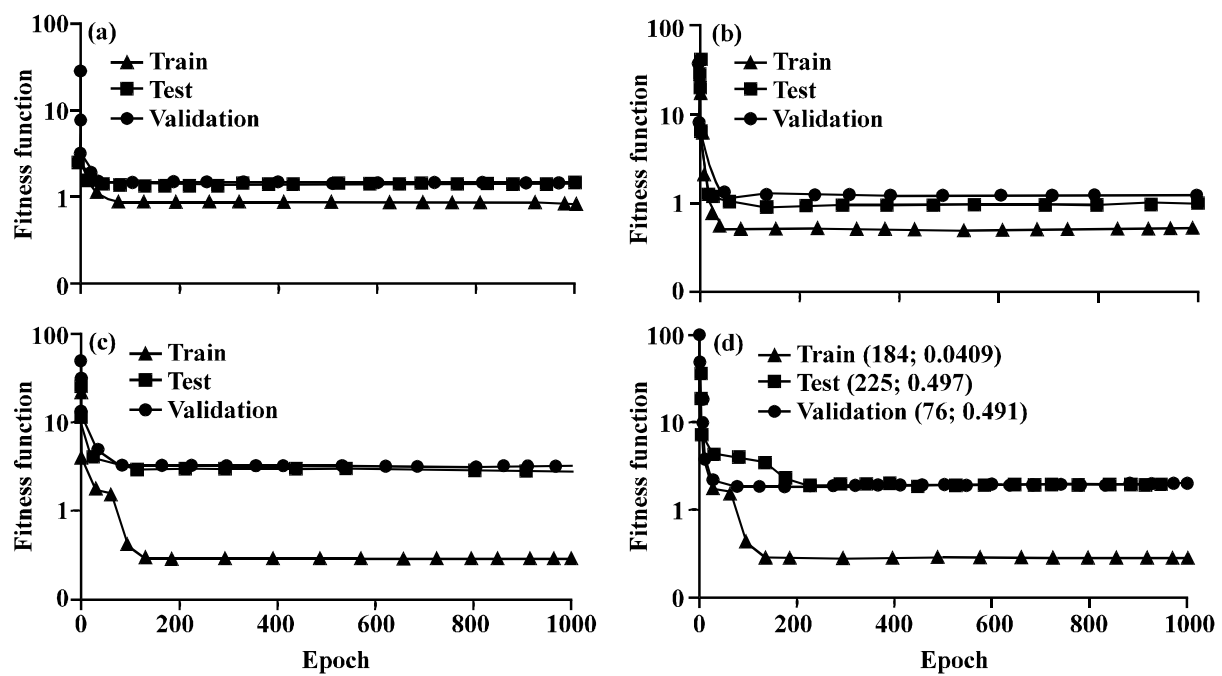

Fig. 5: Fitness function versus epochs for three ANs: a) 5; b) 10; c) 20 and d) 40 neurons in both hidden layers
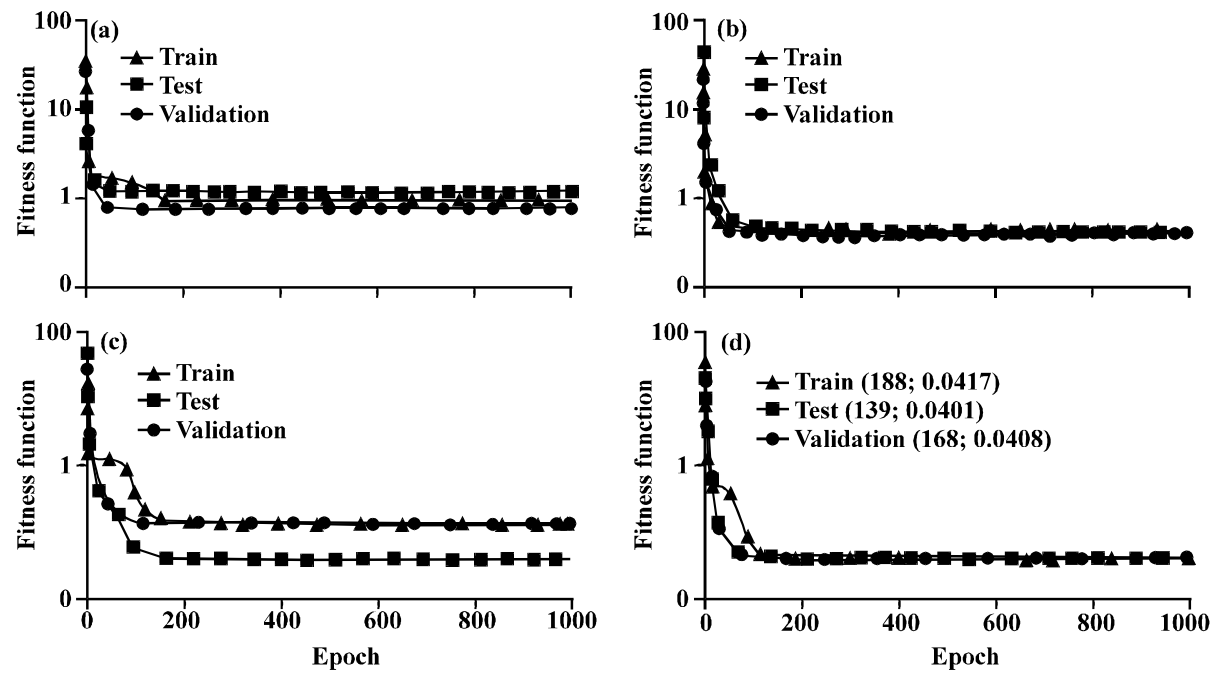

Fig. 6: Fitness function versus epochs for four ANs: a) 5; b) 10; c) 20 and d) 40 neurons in both hidden layers

for 5,10,20 and 40 neurons, respectively, when three ANs were adopted. Figure $6 \mathrm{a}-\mathrm{d}$ present the similar results observed for $5,10,20$ and 40 neurons when four ANs were used, respectively.

Figure 5 shows that a set of 40 neurons offers the best solution for FFNN algorithm wherein the fitness functions of $0.0409,0.497$ and 0.491 were achieved after 184,225 and 76 epochs, respectively, for training, testing and validating the proposed method as shown in Fig. $5 \mathrm{~d}$. Considering the other neurons, the sets of 5, 10 and 20 neurons obtained high error values. However, the group of 40 neurons consumed more time than the sets of 5,10 and 20 neurons. Figure 6 shows that the group of 40 neurons presented the best solution for FFNN algorithm wherein the fitness functions of $0.0417,0.0401$ and 0.0408 were achieved after 188, 139 and 168 epochs, respectively, for training, testing and validating the proposed method as shown in Fig. 6d. Considering the other neurons, the sets of 5,10 and 20 neurons obtained high error values. However, using the group 40 neurons was more time-consuming than the sets of 5,10 and 20 neurons. For testing and validating, the fitness function of the four ANs at 40 neurons improved compared with that of the FFNN that used three ANs. Accordingly, the localization errors were minimized.

Performance of FFNN algorithm: Figure 7 and 8 illustrate the training, testing and validating of FFNN data 

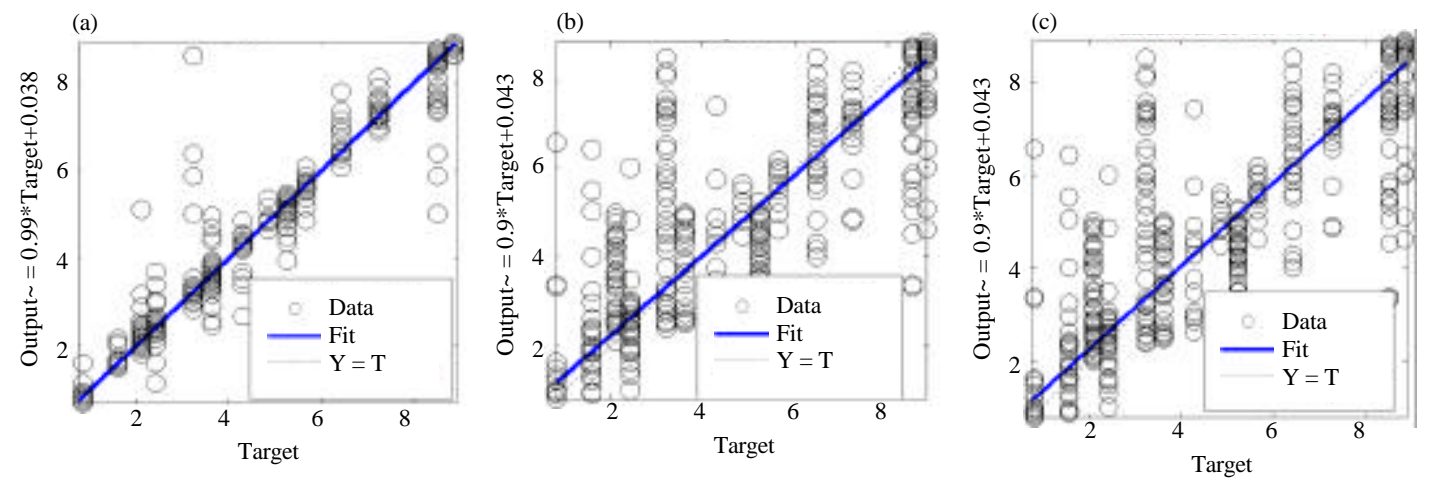

Fig. 7: Performance of FFNN algorithm for: a) Training, $\mathrm{R}=0.9957$; $\mathrm{b}$ ) Testing, $\mathrm{R}=0.95$ and c) Validating, $\mathrm{R}=0.94999$ data using three ANs
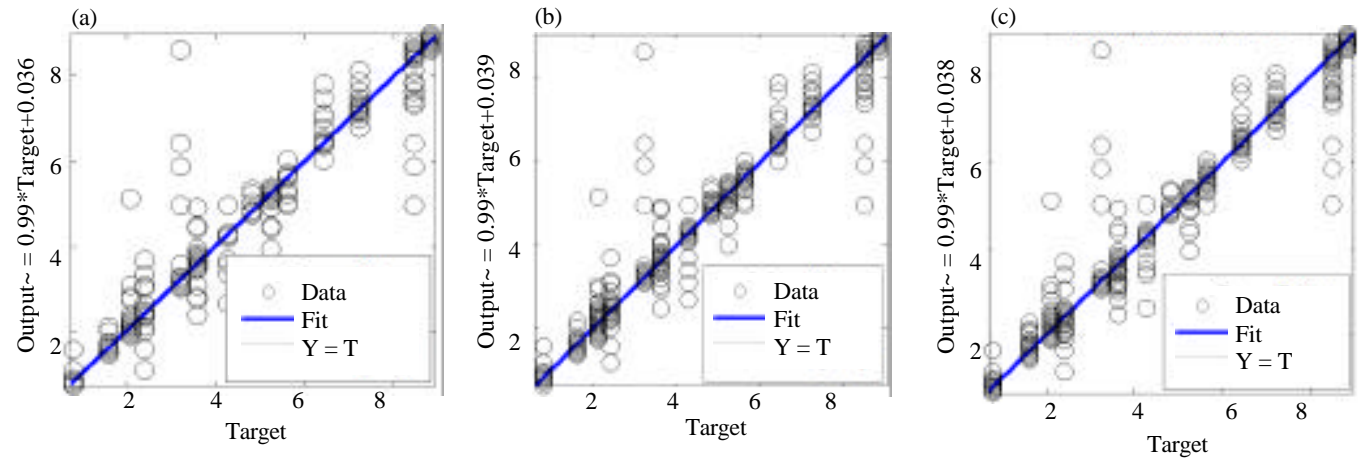

Fig. 8: Performance of FFNN algorithm for: a) Training, $\mathrm{R}=0.99589$; b) Testing, $\mathrm{R}=0.9956$ and (c) Validating, $\mathrm{R}=0.99565$ data using four ANs

for three and four ANs, respectively, when 40 neurons were selected. The actual location (i.e., the target on $\mathrm{x}$-axis) is plotted against the predicted location (i.e., the output on $y$-axis). The correlation coefficient, $R$ between the predicted and actual locations serves as a good determinant for the analysis of prediction performance of FFNN algorithm. Figure 7 shows the R-values for the three ANs used in the first experiment: 0.9957 (training), 0.95 (testing) and 0.9499 (validating). Figure 8 shows the R-values for the four ANs used in the second experiment: 0.9958 (training), 0.9956 (testing) and 0.9956 (validating). The correlation coefficient results revealed a close agreement between the predicted and actual locations. However, the $\mathrm{R}$ performance of the testing and validating data of the four ANs (Fig. 8b) is better than that of the three ANs (Fig. 8c).

Elderly localization estimation: The estimated and actual locations of the MN (elderly people) were investigated for 33 locations in two scenarios. Figure 9a and b show the estimated and actual locations of the MNs for three and four ANs, respectively. The black square points represent the actual locations in meters for 2 coordinates (i.e., $\mathrm{x}$ and $\mathrm{y}$-axis) whereas the red circle points indicate
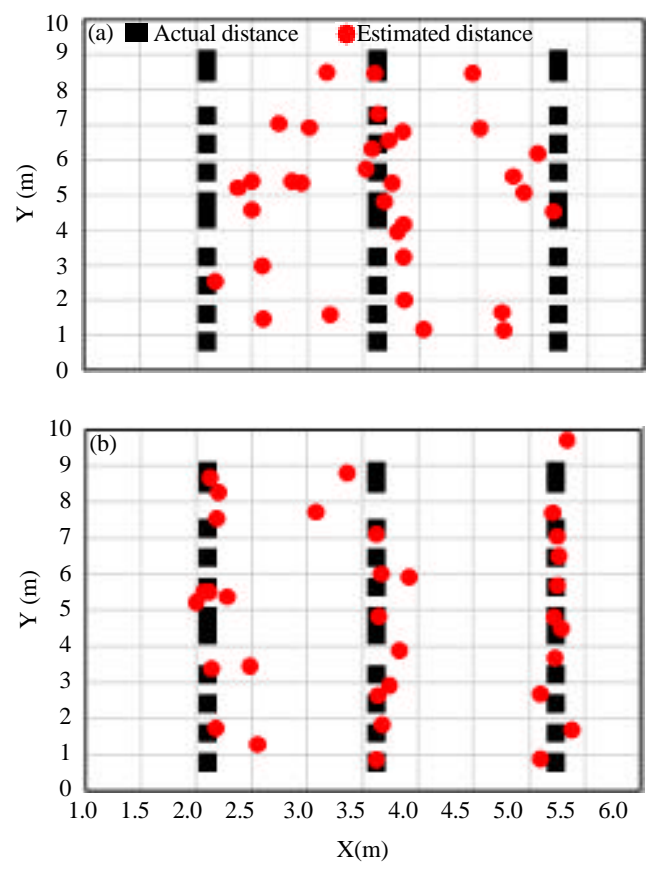

Fig. 9: Actual and predicted elderly locations based on: a) Three and b) Four ANs 


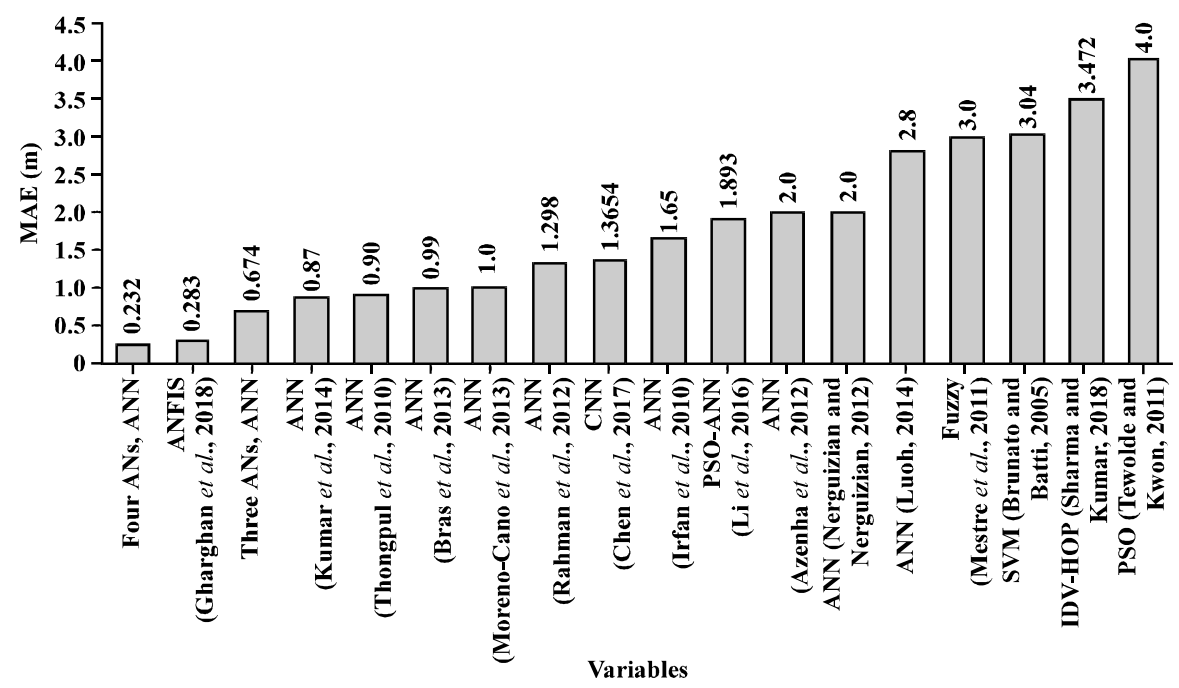

Fig. 10: Comparison of FFNN based on LM with previous works for indoor environments

Table 3: Statistical analyses of the training, testing, and validating data on the basis of three ANs

\begin{tabular}{lccc}
\hline $\begin{array}{l}\text { Type of process/No. of } \\
\text { neurons in each hidden layer }\end{array}$ & MAE (m) & MSE (m) & RMSE (m) \\
\hline FFNN-train & & & \\
5 & 0.712 & 0.947 & 0.973 \\
10 & 0.482 & 0.518 & 0.721 \\
20 & 0.216 & 0.127 & 0.357 \\
40 & 0.051 & 0.014 & 0.203 \\
FFNN-test & & & \\
5 & 0.851 & 1.391 & 1.179 \\
10 & 0.734 & 2.797 & 1.672 \\
20 & 0.684 & 1.194 & 1.092 \\
40 & 0.674 & 1.667 & 1.080 \\
FFNN-validation & & & \\
5 & 0.897 & 1.527 & 1.235 \\
10 & 0.794 & 1.832 & 1.141 \\
20 & 0.725 & 1.246 & 1.116 \\
40 & 0.693 & 1.244 & 1.115 \\
\hline
\end{tabular}

the estimated locations. Figure 9a shows the considerable difference between the actual and predicted locations. However, the differences were minimized and convergence increased between the predicted and actual locations when four ANs were considered as shown in Fig. 9b. The four ANs exhibited higher accuracy than the three ANs. Therefore, we can conclude that the increase in the number of Ans improves localization accuracy (Fig. 10).

Statistical analysis of elderly localization: In this subsection, the results of prediction location for two experiments are investigated in terms of statistical analysis. Three types of statistical analyses were considered to evaluate the accuracy of elderly localization: MAE, Mean Square Error (MSE) and Root MSE (RMSE). These statistical analyses were adopted to prove the results and to show the effect of increased
Table 4: Statistical analyses of the training, testing and validating data on the basis of four ANs

\begin{tabular}{lccc}
\hline $\begin{array}{l}\text { Type of process/No. of } \\
\text { neurons in each hidden layer }\end{array}$ & MAE $(\mathrm{m})$ & MSE (m) & RMSE (m) \\
\hline FFNN-train & & & \\
5 & 0.673 & 0.887 & 0.941 \\
10 & 0.412 & 0.418 & 0.646 \\
20 & 0.195 & 0.117 & 0.342 \\
40 & 0.048 & 0.039 & 0.198 \\
FFNN-test & & & \\
5 & 0.732 & 1.044 & 1.022 \\
10 & 0.588 & 0.929 & 0.964 \\
20 & 0.515 & 1.156 & 1.075 \\
40 & 0.232 & 0.031 & 0.178 \\
FFNN-validation & & & \\
5 & 0.696 & 1.044 & 1.022 \\
10 & 0.461 & 0.575 & 0.758 \\
20 & 0.430 & 0.979 & 0.989 \\
40 & 0.313 & 0.387 & 0.622 \\
\hline
\end{tabular}

number of ANs on localization error. Table 3 and 4 presents the statistical analysis parameters (MAE, MSE and RMSE) obtained on the basis of three and four ANs, respectively. The statistical parameters were computed for 3 FFNN processes (i.e., training, testing and validating). Each process was calculated for 4 different cases of neurons in each hidden layer (i.e., 5, 10, 20 and 40 neurons). Table 3 shows that the elderly localization error is higher for the cases of 5, 10 and 20 neurons compared with that for the case of 40 neurons. However as shown in Table 4, the localization error was minimized to 0.232 (MAE), 0.031 (MSE) and $0.178 \mathrm{~m}$ (RMSE) for the testing data for the case of 40 neurons when four $\mathrm{ANs}$ were used relative to the same parameters and neuron cases when three ANs were employed. The elderly localization error improved when the numbers of neurons and ANs increased. When four ANs and the case of 40 neurons of FFNN were adopted, the MAE of elderly 
localization improved by $6.2 \%$ (training), $65 \%$ (testing) and $54.83 \%$ (validating) compared with those of the three ANs based on Eq. 3:

$$
\text { Localization }_{\text {improv. }}=\left(\frac{\left(\mathrm{MAE}_{3 \mathrm{ANs}}-\mathrm{MAE}_{4 \mathrm{ANs}}\right)}{\mathrm{MAE}_{3 \mathrm{ANs}}}\right) \times 100
$$

Comparison with previous researches: Elderly localization method using three and four ANs was compared with those in previous studies (Fig. 10). The 16 reliable studies (Luoh, 2014; Azenha et al., 2012; Chen et al., 2017; Sharma and Kumar, 2018; Li et al., 2016; Gharghan et al., 2018; Thongpul et al., 2010; Nerguizian and Nerguizian, 2007; Brunato and Battiti, 2005; Kumar et al., 2014; Bras et al., 2013; Moreno-Cano et al., 2013; Rahman et al., 2012; Irfan et al., 2010; Mestre et al., 2011; Tewolde and Kwon, 2011) were compared with our work in terms of the MAE of elderly localization. The comparison was conducted to validate and support our adopted FFNN-based RSSIs of the ANs. Most the studies adopted artificial intelligence techniques for localization purposes. In addition, the afore mentioned previous works localized the nodes on the basis of the RSSIs of Zigbee WSN in indoor environments. The comparison results indicate that elderly localization based on FFNN using three and four ANs is superior to that of other methods in previous studies with MAE reaching 0.674 (three $\mathrm{ANs}$ ) and $0.232 \mathrm{~m}$ (four ANs) when using 40 neurons (Fig. 10).

\section{CONCLUSION}

This study presented an elderly localization method using a combination of the FFNN-based LM algorithm and RSSIs of Zigbee ANs. Two experiments were conducted in the Electronic Lab in the Laboratories of the Electrical Engineering Technical College. The first experiment adopted three ANs distributed inside the testing area of the lab whereas the second experiment adopted four ANs. Two hidden layers were used in training, testing and validating the FFNN. Four sets of neurons $(5,10,20$ and 40) for each hidden layer were examined to evaluate the elderly localization accuracy. The results show that when the number of $\mathrm{ANs}$ and neurons increased, the elderly localization accuracy can be improved. In terms of statistical analysis, the localization accuracy based on four ANs is better than that based on three ANs for all the cases of adopted neurons. The comparison results also show that our proposed method outperforms other studies in terms of MAE. Future researches will focus on the practical implementation of FFNN on the basis of the obtained RSSIs in real time. In addition, other hybrid techniques that use FFNN and optimization algorithm can be evaluated to further improve the elderly localization accuracy.

\section{ACKNOWLEDGEMENTS}

The researchers would like to thank the staff of the Department of Medical Instrumentation Techniques Engineering, College of Electrical Engineering Techniques, Middle Technical University for their support to this research.

\section{REFERENCES}

Azenha, A., L. Peneda and A. Carvalho, 2012. A neural network approach for radio frequency based indoors localization. Proceedings of the IECON 38th 2012 Annual International Conference on IEEE Industrial Electronics Society, October 25-28, 2012, IEEE, Montreal, Canada, ISBN:978-1-4673-2419-9, pp: 5990-5995.

Blumrosen, G., B. Hod, T. Anker, D. Dolev and B. Rubinsky, 2013. Enhancing RSSI-based tracking accuracy in wireless sensor networks. ACM. Trans. Sens. Networks, 9: 1-29.

Bras, L., P. Pinho and N.B. Carvaloh, 2013. Evaluation of a sectorised antenna in an indoor localisation system. IET. Microwaves Antennas Propag., 7: 679-685.

Brunato, M. and R. Battiti, 2005. Statistical learning theory for location fingerprinting in wireless LANs. Comput. Networks, 47: 825-845.

Chen, H., Y. Zhang, W. Li, X. Tao and P. Zhang, 2017. ConFi: Convolutional neural networks based indoor wiFi localization using channel state information. IEEE. Access, 5: 18066-18074.

Cheng, L., C, Wu, Y. Zhang, H. Wu, M. Li and C. Maple, 2012. A survey of localization in wireless sensor network. Intl. J. Distrib. Sens. Networks, 8: $1-12$.

Erazo-Rodas, M., M. Sandoval-Moreno, S. Munoz-Romero, M. Huerta and D. Rivas-Lalaleo et al., 2018. Multiparametric monitoring in equatorian tomato greenhouses (I): Wireless sensor network benchmarking. Sens., 18: 25-55.

Gharghan, S., R. Nordin and M. Ismail, 2016a. A wireless sensor network with soft computing localization techniques for track cycling applications. Sens., 16: $1-23$.

Gharghan, S.K., R. Nordin, A.M. Jawad, H.M. Jawad and M. Ismail, 2018. Adaptive neural fuzzy inference system for accurate localization of wireless sensor network in outdoor and indoor cycling applications. IEEE. Access, 6: 38475-38489.

Gharghan, S.K., R. Nordin, M. Ismail and J.A. Ali, 2016 b. Accurate wireless sensor localization technique based on hybrid PSO-ANN algorithm for indoor and outdoor track cycling. IEEE. Sens. J., 16: 529-541. 
Gogolak, L., S. Pletl and D. Kukolj, 2013. Neural network-based indoor localization in WSN environments. Acta Polytech. Hungarica, 10: 221-235.

Halder, S. and A. Ghosal, 2016. A survey on mobility-assisted localization techniques in wireless sensor networks. J. Network Comput. Appl., 60: 82-94.

Hevrdejs, K., J. Knoll and S. Miah, 2017. A Zigbee-based framework for approximating sensor range and bearing. Proceedimgs of the 2017 IEEE 30th Canadian Conference on Electrical and Computer Engineering (CCECE), April 30- May 3, 2017, IEEE, Windsor, Canada, ISBN:978-1-5090-5539-5, pp: 1-4.

Hwang, R.C., P.T. Hsu, J. Cheng, C.Y. Chen and C.Y. Chang et al., 2011. The indoor positioning technique based on neural networks. Proceedings of the 2011 IEEE International Conference on Signal Processing, Communications and Computing (ICSPCC), September 14-16, 2011, IEEE, Xi'an, China, ISBN:978-1-4577-0893-0, pp: 1-4.

Irfan, N., M. Bolic, M.C. Yagoub and V. Narasimhan, 2010. Neural-based approach for localization of sensors in indoor environment. Telecommun. Syst., 44: 149-158.

Karim, L., N. Nasser, Q.H. Mahmoud, A. Anpalagan and T.E. Salti, 2015. Range-free localization approach for M2M communication system using mobile anchor nodes. J. Network Comput. Appl., 47: 137-146.

Kaur, A., P. Kumar and G.P. Gupta, 2017. A weighted centroid localization algorithm for randomly deployed wireless sensor networks. J. King Saud Univ. Comput. Inf. Sci., 31: 82-91.

Kumar, S., S.M. Jeon and S.R. Lee, 2014. Localization estimation using artificial intelligence technique in wireless sensor networks. Korean Inst. Commun. Sci., 39: 820-827.

Li, N., J. Chen, Y. Yuan, X. Tian and Y. Han et al., 2016. A Wi-Fi indoor localization strategy using particle swarm optimization based artificial neural networks. Intl. J. Distrib. Sens. Networks, 12: 1-9.

Lin, C.M., Y.J. Mon, C.H. Lee, J.G. Juang and I.J. Rudas, 2014. ANFIS-based indoor location awareness system for the position monitoring of patients. Acta Polytech. Hung., 11: 37-48.

Liu, T., X. Luo and Z. Liang, 2018. Enhanced sparse representation-based device-free localization with radio tomography networks. J. Sens. Actuator Networks, 7: 1-16.

Lo, G., S. Gonzalez-Valenzuela and V.C. Leung, 2013. Wireless body area network node localization using small-scale spatial information. IEEE. J. Biomed. Health Inf., 17: 715-726.

Luoh, L., 2014. Zigbee-based intelligent indoor positioning system soft computing. Soft Comput., 18: 443-456.
Mahapatra, R.K. and N.S.V. Shet, 2018. Localization based on RSSI exploiting gaussian and averaging filter in wireless sensor network. Arabian J. Sci. Eng., 43: 4145-4159.

Mestre, P., L. Coutinho, L. Reigoto, J. Matias and A. Correia et al., 2011. Indoor Location using Fingerprinting and Fuzzy Logic. In: Eurofuse 2011, Advances in Intelligent and Soft Computing, Melo-Pinto, P., P. Couto, C. Serodio, J. Fodor and B. De Baets (Eds.). Springer, Berlin, Heidelberg, Germany, ISBN:978-3-642-24000-3, pp: 363-374.

Moreno-Cano, M.V., M.A. Zamora-Izquierdo, J. Santa and A.F. Skarmeta, 2013. An indoor localization system based on artificial neural networks and particle filters applied to intelligent buildings. Neurocomputing, 122: $116-125$.

Nerguizian, C. and V. Nerguizian, 2007. Indoor fingerprinting geolocation using wavelet-based features extracted from the channel impulse response in conjunction with an artificial neural network. Proceedings of the 2007 IEEE International Symposium on Industrial Electronics, June 4-7, 2007, IEEE, Vigo, Spain, ISBN:978-1-4244-0754-5, pp: 2028-2032.

Ostlin, E., H.J. Zepernick and H. Suzuki, 2010. Macrocell path-loss prediction using artificial neural networks. IEEE. Trans. Veh. Technol., 59: 2735-2747.

Pak, J.M., C.K. Ahn, P. Shi, Y.S. Shmaliy and M.T. Lim, 2017. Distributed hybrid particle/FIR filtering for mitigating NLOS effects in TOA-based localization using wireless sensor networks. IEEE. Trans. Ind. Electron., 64: 5182-5191.

Paul, A. and T. Sato, 2017. Localization in wireless sensor networks: A survey on algorithms, measurement techniques, applications and challenges. J. Sens. Actuator Networks, 6: 1-23.

Payal, A., C.S. Rai and B.R. Reddy, 2015. Analysis of some feedforward artificial neural network training algorithms for developing localization framework in wireless sensor networks. Wireless Pers. Commun., 82: 2519-2536.

Perera, R., A. Perez, M. Garcia-Dieguez and J. Zapico-Valle, 2017. Active wireless system for structural health monitoring applications. Sens., 17: $1-16$.

Pradityo, H.P., L. Rosyidi and R.F. Sari, 2017. Performance evaluation of RSS fingerprinting method to track Zigbee devices location using artificial neural networks. Proceedings of the 2017 International Conference on Information and Communication Technology Convergence (ICTC), October 18-20, 2017, IEEE, Jeju, South Korea, ISBN: 978-1-5090-4033-9, pp: 268-273. 
Rahman, M.S., Y. Park and K.D. Kim, 2012. RSS-Based indoor localization algorithm for wireless sensor network using generalized regression neural network. Arab. J. Sci. Eng., 37: 1043-1053.

Ramiro Martinez-de Dios, J., A. Ollero, F. Fernandez and C. Regoli, 2017. On-line RSSI-range model learning for target localization and tracking. J. Sens. Actuator Networks, 6: 1-15.

Redondi, A., M. Chirico, L. Borsani, M. Cesana and M. Tagliasacchi, 2013. An integrated system based on wireless sensor networks for patient monitoring, localization and tracking. Ad Hoc Networks, 11: 39-53.

Sharma, G. and A. Kumar, 2018. Improved DV-Hop localization algorithm using teaching learning based optimization for wireless sensor networks. Telecommun. Syst., 67: 163-178.

Tewolde, G.S. and J. Kwon, 2011. Efficient WiFi-based indoor localization using particle swarm optimization. Proceedings of the 2nd 2011 International Conference on Swarm Intelligence, June 12-15, 2011 , Chongqing, China, ISBN:978-3-642-21514-8, pp: 203-211.

Thongpul, K., N. Jindapetch and W. Teerapakajorndet, 2010. A neural network based optimization for wireless sensor node position estimation in industrial environments. Proceedings of the 2010 ECTI International Confernce on Electrical Engineering/Electronics, Computer, Telecommunications and Information Technology ECTI-CON2010, May 19-21, 2010, EEEE, Chiang Mai, Thailand, ISBN: 978-1-4244-5606-2, pp: 249-253.

Tomic, S., M. Beko and R. Dinis, 2017. 3-D target localization in wireless sensor networks using RSS and AoA measurements. IEEE. Trans. Veh. Technol., 66: 3197-3210.

Velimirovic, A.S., G.L. Djordjevic, M.M. Velimirovic and M.D. Jovanovic, 2012. Fuzzy Ring-Overlapping Range-Free (FRORF) localization method for wireless sensor networks. Comput. Commun., 35: 1590-1600.
Wang, X., L. Gao, S. Mao and S. Pandey, 2017. CSI-based fingerprinting for indoor localization: A deep learning approach. IEEE. Trans. Veh. Technol., 66: 763-776.

Wang, Y. and Y. Wu, 2017. An efficient semidefinite relaxation algorithm for moving source localization using TDOA and FDOA measurements. IEEE. Commun. Lett., 21: 80-83.

Wilamowski, B.M. and H. Yu, 2010. Improved computation for levenberg-marquardt training. IEEE. Trans. Neural Networks, 21: 930-937.

Zhang, T., J. He and Y. Zhang, 2012. Secure sensor localization in wireless sensor networks based on neural network. Intl. J. Comput. Intell. Syst., 5: 914-923.

Zhao, J., W. Xi, Y. He, Y. Liu and X.Y. Li et al., 2013. Localization of wireless sensor networks in the wild: Pursuit of ranging quality. IEEE. ACM. Trans. Networking, 21: 311-323.

Zhao, L.Z., X.B. Wen and D. Li, 2014. Amorphous localization algorithm based on BP artificial neural network. Proceedings of the 2014 International Conference on Software Intelligence Technologies and Applications Frontiers of Internet of Things, December 4-6, 2014, Hsinchu, Taiwan, ISBN:978-1-84919-970-4, pp: 178-183.

Zhao, Y. and N. Patwari, 2016. An experimental comparison of radio transceiver and transceiver-free localization methods. J. Sens. Actuator Networks, 5: 1-11.

Zhi-kun, L. and L. Zhong, 2015. Node self-localization algorithm for wireless sensor networks based on modified particle swarm optimization. Proceedings of the 27th Chinese Conference on Control and Decision (2015 CCDC), May 23-25, 2015, IEEE, Qingdao, China, pp: 5968-5971. 